Supporting Information

\title{
Massively Parallel GPU-Accelerated String Method for Fast and Accurate Prediction of Molecular Diffusivity in Nanoporous Materials
}

\author{
Musen Zhou and Jianzhong $\mathrm{Wu}$ \\ Department of Chemical and Environmental Engineering, University of California, Riverside, \\ Riverside, CA, 92521, United States
}

The supporting information provides the definitions of rotational variables and the full details of the force-field parameters for all polyatomic molecules investigated in the work. It also provides the memory usage for different GPU paralleled implementations of the string method and additional figures of molecular structures of p-type and o-type xylene in MOF-5. In addition, it presents the structural properties and diffusion coefficients for MOFs with the highest diffusion selectivity for ethane/ethene separation. Movies from different viewpoint of string evolution for ethene in MOF-5 are available in the format of MPG.

\section{Definition of Molecular Orientation}

As shown in Figure S1, the orientation of a rigid molecule such as ethene would be a function of the three Euler angles, $\alpha, \beta, \gamma$, relative to the material frame.

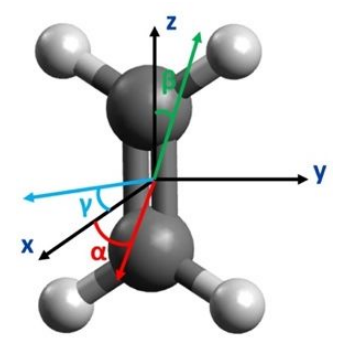

Figure S1. Definition of orientation variables (viz., Euler angles) for an ethene molecule relative to the material frame. 


\section{Force Fields}

For direct comparison with simulation data, we use the force field parameters for polyatomic molecules and nanoporous materials the same as those used in molecular dynamics simulations. For most polyatomic molecules considered in this work, the simulation results were based on the united atom force field (TraPPE-UA) without electrostatic interactions. ${ }^{1,2}$ For nitrogen and carbon dioxide, each atom is modeled as a single Lennard-Jones (LJ) site with the point charge the same as that used in the TraPPE force field. ${ }^{3}$ To balance the charge neutrality, a positive charge is placed in the center of nitrogen molecule. The DFT-derived force field is used for benzene. ${ }^{4}$ Table S1 shows the force-field parameters for all molecules considered in this work.

Table S1. LJ parameters and partial charge for polyatomic molecules considered in this work

\begin{tabular}{cccc}
\hline & $\sigma(\AA)$ & $\varepsilon / \mathrm{k}_{\mathrm{B}}(\mathrm{K})$ & charge $(e)$ \\
\hline $\mathrm{CH}_{4}$ (methane) & 3.730 & 148 & \\
$\mathrm{CH}_{3}$ - (ethane) & 3.760 & 108 & \\
$\mathrm{CH}_{2}$ - (ethene) & 3.680 & 92.8 & \\
$\mathrm{C}$ - (xylene) & 3.850 & 20.0 & \\
$\mathrm{CH}$ - (xylene) & 3.695 & 50.5 & \\
$\mathrm{CH}_{3}$ - (xylene) & 3.750 & 98.0 & \\
$\mathrm{C}$ - (benzene) & 3.470 & 47.81 & -0.15 \\
$\mathrm{H}-$ (benzene) & 2.850 & 7.55 & 0.15 \\
$\mathrm{C}-$ (carbon dioxide) & 2.80 & 27 & 0.4 \\
$\mathrm{O}-$ (carbon dioxide) & 3.05 & 79 & -0.2 \\
$\mathrm{~N}-$ (nitrogen) & 3.31 & 36 & -0.482 \\
$\mathrm{COM}$ (nitrogen) & 0 & 0 & 0.964 \\
\hline
\end{tabular}

\section{Memory Usage for Different GPU Implementations}

The memory usage for different GPU paralleled implementations of the string method versus the number of images on the reaction coordinate (viz., string) for ethene in MOF-5 is shown in Figure S2. For the Nvidia Tesla P100 graphic card used in this work, 16 GB memory is available for data allocation while Intel Xeon E5-2640 CPU has 64 GB physical memory. According to 
Figure S2, massive GPU paralleled implementation uses much more memory than simple GPU paralleled implementation because it requires more auxiliary variables during the external potential calculation. With 16 GB memory on Nvidia Tesla P100, up to 5,000 images can be allocated for ethene in MOF-5 with massive GPU parallel implementation while more than 10,000,000 images can be allocated for simple GPU parallel implementation.

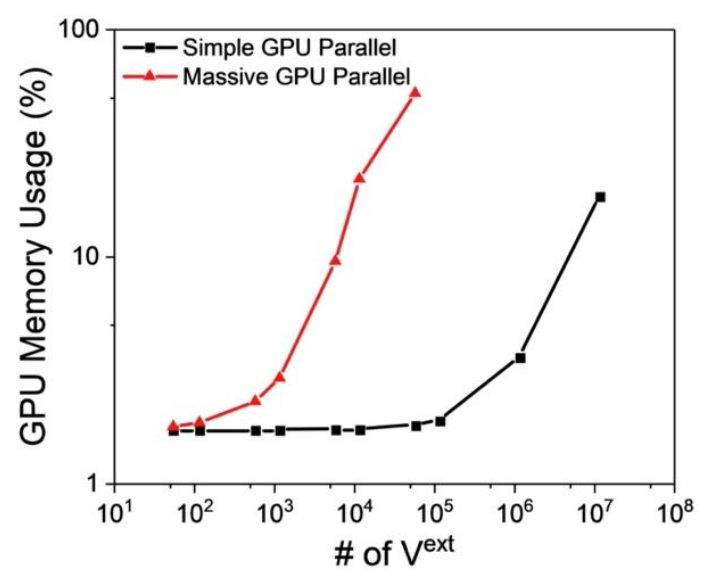

Figure S2. Percentage of GPU memory usage versus the number of $V^{e x t}$ calculations for ethene diffusion in MOF-5.

\section{P-type and O-type Xylene in MOF-5}

Figure S3 shows the positions and orientations of p-type and o-type xylene molecules across the pore center in MOF-5 when they are moving along the specific minimum-energy paths. We see that the center of mass for p-type xylene is closer to the pore center in MOF-5 than that for otype xylene, while o-type xylene, especially its methyl group, is much closer to the metal cluster. 

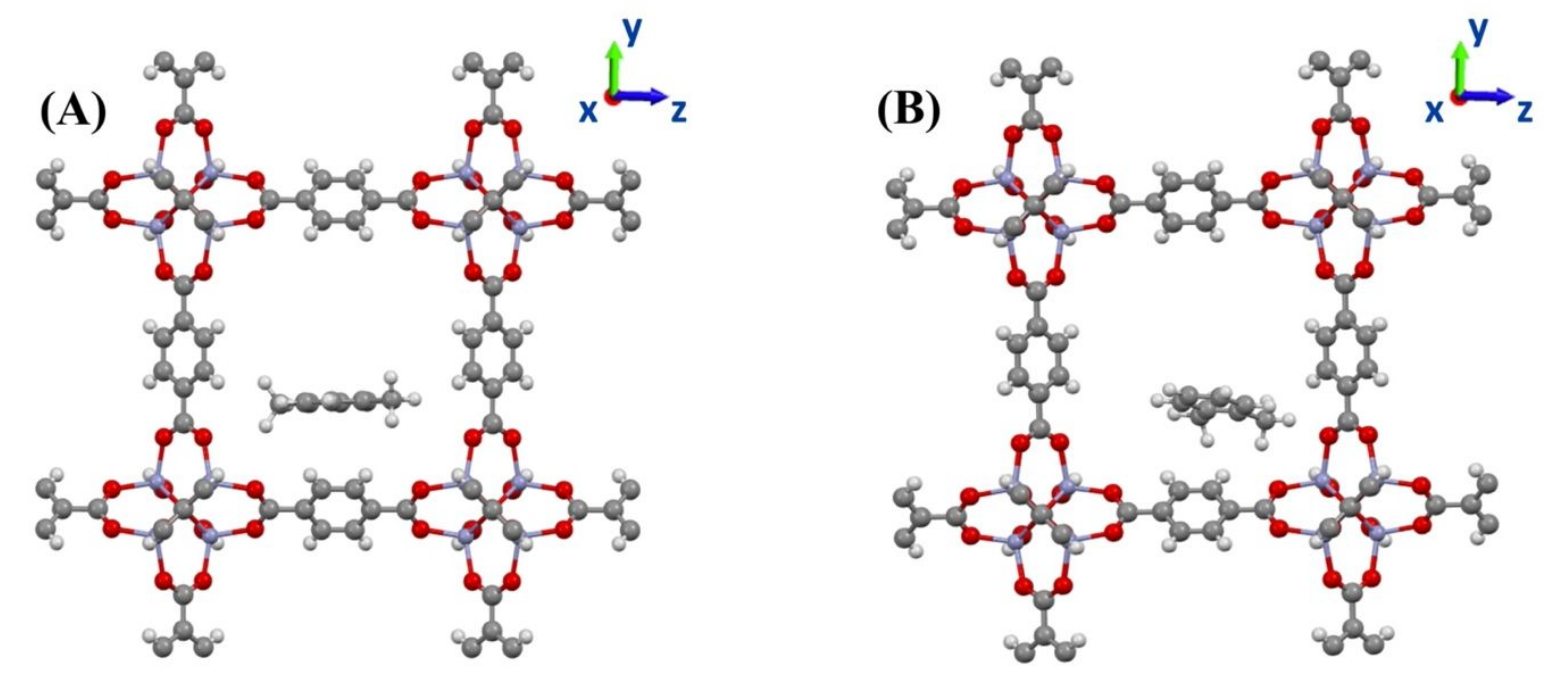

Figure S3. Molecular position and orientation of p-type xylene (A) and o-type xylene (B) in MOF5 when hopping across pore (along x direction) along the minimum energy path. (P-type and otype xylene are modeled as eight united group sites according to TraPPE-UA force field. The detailed guest molecular structures are for illustration purpose only).

\section{MOFs with the Highest Diffusion Selectivity for Ethane/Ethene Separation}

Table S2. Diffusion coefficients and structural properties of MOFs with the highest diffusion selectivity for ethane/ethene separation

\begin{tabular}{ccccccc}
\hline Ref Code & $S_{\text {diff, } C_{2} H_{4} / C_{2} H_{6}}$ & $\begin{array}{c}\text { PLD } \\
(\AA)\end{array}$ & $\begin{array}{c}\text { LCD } \\
(\AA)\end{array}$ & $\begin{array}{c}\text { void } \\
\text { fraction }\end{array}$ & $\begin{array}{c}D_{C_{2} H_{4}} \\
\left(\mathrm{~m}^{2} / \mathrm{s}\right)\end{array}$ & $\begin{array}{c}D_{C_{2} H_{6}} \\
\left(\mathrm{~m}^{2} / \mathrm{s}\right)\end{array}$ \\
\hline YIGFIF & 57.69 & 3.376 & 5.022 & 0.521 & $2.12 \times 10^{-11}$ & $3.68 \times 10^{-13}$ \\
SUPSIG02 & 53.67 & 3.883 & 5.892 & 0.522 & $2.41 \times 10^{-12}$ & $4.50 \times 10^{-14}$ \\
OGIBOV & 50.00 & 3.663 & 4.920 & 0.428 & $1.45 \times 10^{-12}$ & $2.90 \times 10^{-14}$ \\
WIXRUQ & 37.87 & 3.644 & 5.220 & 0.400 & $4.09 \times 10^{-12}$ & $1.08 \times 10^{-13}$ \\
YAQZEX & 34.22 & 3.356 & 5.113 & 0.436 & $9.17 \times 10^{-11}$ & $2.68 \times 10^{-12}$ \\
WUNTUV & 28.85 & 3.469 & 5.530 & 0.476 & $4.27 \times 10^{-13}$ & $1.48 \times 10^{-14}$ \\
WEDXAG & 24.81 & 3.751 & 5.270 & 0.374 & $2.58 \times 10^{-12}$ & $1.04 \times 10^{-13}$ \\
GAWCUF & 24.17 & 3.203 & 5.312 & 0.461 & $3.19 \times 10^{-13}$ & $1.32 \times 10^{-14}$ \\
YOFXEY & 23.86 & 3.801 & 5.346 & 0.463 & $9.21 \times 10^{-12}$ & $3.86 \times 10^{-13}$ \\
TEWFOS & 23.64 & 3.413 & 5.270 & 0.449 & $1.17 \times 10^{-11}$ & $4.95 \times 10^{-13}$ \\
\hline
\end{tabular}




\section{Videos on String evolution}

We provide two videos from different viewpoints to illustrate how the string method attains the minimum energy path for the diffusion of a polyatomic molecule in nanoporous material. These videos were made from the visual molecular dynamics (VMD) package based on the evolution of the string for ethene in MOF-5. ${ }^{5}$ Although in this work ethene is modeled as a diatomic molecule according to the TraPPE-UA force field, the detailed atomistic structure is shown only for the sake of illustration. In every frame of the video, the ethene molecule has a fixed position and orientation as represented by one image along the string. In each video, only 11 images are displayed for clarity. We see that not only the shape of the string, but also the orientation of each image, changes during the string evolution in order to achieve the minimum energy path. In the final frame of each video, the string depicts how molecular position and orientation change along the minimum energy path for ethene diffusion in MOF-5.

\section{References}

1. Wu, Y.; Chen, H.; Liu, D.; Qian, Y.; Xi, H., Adsorption and separation of ethane/ethylene on ZIFs with various topologies: Combining GCMC simulation with the ideal adsorbed solution theory (IAST). Chemical Engineering Science 2015, 124, 144-153.

2. Wick, C. D.; Martin, M. G.; Siepmann, J. I., Transferable potentials for phase equilibria. 4. United-atom description of linear and branched alkenes and alkylbenzenes. The Journal of Physical Chemistry B 2000, 104 (33), 8008-8016.

3. Potoff, J. J.; Siepmann, J. I., Vapor-liquid equilibria of mixtures containing alkanes, carbon dioxide, and nitrogen. AIChE journal 2001, 47 (7), 1676-1682.

4. Amirjalayer, S.; Schmid, R., Adsorption of Hydrocarbons in Metal-Organic Frameworks: A Force Field Benchmark on the Example of Benzene in Metal-Organic Framework 5. The Journal of Physical Chemistry C 2012, 116 (29), 15369-15377.

5. Humphrey, W.; Dalke, A.; Schulten, K., VMD: visual molecular dynamics. Journal of molecular graphics 1996, 14 (1), 33-38. 